\title{
DECONbench: a benchmarking platform dedicated to deconvolution methods for tumor heterogeneity quantification
}

\author{
Clémentine Decamps ${ }^{1 \dagger}$, Alexis Arnaud ${ }^{2 \dagger}$, Florent Petitprez ${ }^{3}$, Mira Ayadi $^{3}$, Aurélia Baurès ${ }^{3}$, Lucile Armenoult ${ }^{3}$, \\ HADACA consortium, Sergio Escalera ${ }^{4}$, Isabelle Guyon ${ }^{5}$, Rémy Nicolle ${ }^{3}$, Richard Tomasini ${ }^{6}$, Aurélien de Reyniès ${ }^{3}$, \\ Jérôme $\mathrm{Cros}^{7}$, Yuna Blum ${ }^{3,8^{*}}$ (D) and Magali Richard ${ }^{1 *+}$
}

${ }^{*}$ Correspondence:

yuna.blum@univ-rennes1.

fr; magali.richard@univ-

grenoble-alpes.fr

${ }^{\dagger}$ Clémentine Decamps

and Alexis Arnaud should

be regarded as joint First

Authors

†Yuna Blum and Magali

Richard should be regarded

as joint Last Authors

1 Laboratory TIMC-IMAG,

UMR 5525, CNRS, Univ.

Grenoble Alpes, Grenoble,

France ${ }^{8}$ IGDR UMR 6290,

CNRS, Université de Rennes

1, Rennes, France

Full list of author information is available at the end of the article

A full list of Consortium members and their affiliations is available at the end of the text.

\begin{abstract}
Background: Quantification of tumor heterogeneity is essential to better understand cancer progression and to adapt therapeutic treatments to patient specificities. Bioinformatic tools to assess the different cell populations from single-omic datasets as bulk transcriptome or methylome samples have been recently developed, including reference-based and reference-free methods. Improved methods using multi-omic datasets are yet to be developed in the future and the community would need systematic tools to perform a comparative evaluation of these algorithms on controlled data.
\end{abstract}

Results: We present DECONbench, a standardized unbiased benchmarking resource, applied to the evaluation of computational methods quantifying cell-type heterogeneity in cancer. DECONbench includes gold standard simulated benchmark datasets, consisting of transcriptome and methylome profiles mimicking pancreatic adenocarcinoma molecular heterogeneity, and a set of baseline deconvolution methods (reference-free algorithms inferring cell-type proportions). DECONbench performs a systematic performance evaluation of each new methodological contribution and provides the possibility to publicly share source code and scoring.

Conclusion: DECONbench allows continuous submission of new methods in a userfriendly fashion, each novel contribution being automatically compared to the reference baseline methods, which enables crowdsourced benchmarking. DECONbench is designed to serve as a reference platform for the benchmarking of deconvolution methods in the evaluation of cancer heterogeneity. We believe it will contribute to leverage the benchmarking practices in the biomedical and life science communities. DECONbench is hosted on the open source Codalab competition platform. It is freely available at: https://competitions.codalab.org/competitions/27453.

Keywords: Benchmarking platform, Deconvolution, Transcriptome, DNA methylation, Omics integration, Cellular heterogeneity, Cancer author(s) and the source, provide a link to the Creative Commons licence, and indicate if changes were made. The images or other third party material in this article are included in the article's Creative Commons licence, unless indicated otherwise in a credit line to the material. If material is not included in the article's Creative Commons licence and your intended use is not permitted by statutory regulation or exceeds the permitted use, you will need to obtain permission directly from the copyright holder. To view a copy of this licence, visit http:// creativecommons.org/licenses/by/4.0/. The Creative Commons Public Domain Dedication waiver (http://creativecommons.org/publi cdomain/zero/1.0/) applies to the data made available in this article, unless otherwise stated in a credit line to the data. 


\section{Background}

The recent development of high-throughput sequencing technologies has enabled the characterization of the genetic regulations underlying diseases such as cancer. Important advances have been made but studies often overlook the fact that tumors are made up of cells from different identities and origins. The quantification of tumor heterogeneity is of great interest to the biomedical research community because the various components of a tumor are key factors in tumor progression, clinical outcome and response to therapy. To isolate a cell population of interest, microdissection techniques can be performed on clinically heterogeneous tissue samples, but these advanced techniques are not feasible in clinical routine. In addition, single-cell technologies, while promising, have intensive protocols and require expensive and specialized resources, currently hindering their establishment in a clinical setting [1]. Instead, deconvolution methods can be used to infer cell-type composition in silico from bulk measurements, which enable the analysis of a large number of publicly available omic datasets. Bioinformatics tools that assess the different cell populations from bulk transcriptome [2-5] and methylome [6-9] samples have been recently developed, including reference-based and reference-free methods.

Recent efforts have been made to objectively compare existing tools in order to guide the users. In particular, two recent benchmark studies proposed a comprehensive comparison of transcriptome-based deconvolution methods using various parameters and simulation settings $[10,11]$. In the same vein, the DREAM challenge proposed in 2019 [12] a data challenge dedicated to the prediction of immune cell types, showing the emerging spirit towards reproducibility and benchmarking. Although interesting, all these efforts are time-bound and cannot take into account upcoming novel methods. Moreover, the possibility to integrate different types of omic data to infer cell-type proportions is currently under-studied.

Standardized unbiased benchmarking resources are essential to evaluate the performances of computational methods. Indeed, these resources should avoid falling into the 'self-assessment trap', in which researchers are unrealistically expected to fairly compare their own computational method with other similar algorithms [13, 14]. In addition, unbiased attempts to benchmark computational methods are often static in space and time, preventing further contributions of other scientists or the assessment of new methods developed after the publication of the benchmark [15]. Recent collective initiatives provided formal guidelines and unified frameworks to improve unbiased performance evaluation [16]. For instance, the Global Alliance for Genomic and Health (GA4GH) published an open access benchmarking tool to assess germline small variant calls in human genomes [17]. More recently, BEELINE, a uniform interface to evaluate Gene Regulatory Network inference from single-cell data, was published and made freely accessible in the form of a docker image [18].

In this project, we built on a previous HADACA (Health Data Challenge consortium) benchmarking study [7] to develop a standardized benchmark framework for accurately evaluating quantification of tumor intra-heterogeneity from a multi-omic dataset. First, we built in silico 10 paired methylome and transcriptome benchmark datasets, using pancreatic cancer (PDAC, pancreatic adenocarcinoma) as a case study. These benchmark datasets were made realistic by the integration of the latest knowledge on PDAC biology [19-21] in the simulation models and can be used as 'truth' to evaluate computational 
methods quantifying tumor heterogeneity. Second, we defined Mean Absolute Error (MAE) on estimated cell-type proportions and computational time as standard performance metrics. Third, we embedded the benchmark dataset and the scoring algorithm into a web platform called DECONbench. This web platform enables continuous and crowdsourced benchmarking, by asking participants to submit source code of their algorithm. Each submission is therefore run by the platform on the benchmark dataset and results generated in a reproducible way. Fourth, we implemented on the platform baseline methods based on some previously published deconvolution algorithms and tools. Therefore, DECONbench is an open resource to evaluate novel computational methods in an unbiased way. It provides a private general report on the overall performances of the method submitted by any participant and offers the possibility to share all source code of the contributing methods, as well as performance evaluation on a public leaderboard.

Here we present DECONbench, an innovative public benchmarking platform, open source and freely available, aiming at comparing integrative deconvolution methods for tumor heterogeneity quantification. This framework supports both crowdsourcing benchmarking (collaborative and competitive assessment of the methods) and continuous benchmarking (possibility to continuously integrate novel methods), two features that should contribute to the widespread community adoption of benchmarking good practices $[15,22]$. To conclude, DECONbench is an open online benchmark framework including gold standard benchmarking datasets from different types of omic data, stateof-the-art baseline computational methods and it enables the submission of new methods for evaluation.

\section{Implementation}

\section{The benchmarking platform infrastructure}

DECONbench takes advantage of the Codalab web-based platform (https://competitio ns.codalab.org/) to provide a software environment for evaluating deconvolution methods. Users submit a full program that is applied to the provided benchmark datasets and compared to the ground truth. DECONbench outputs a performance score displayed on the leaderboard (Fig. 1).

\section{Usage}

DECONbench is optimized to execute methods developed in R statistical programming language, using a docker image provided on our website. The benchmark is structured around an ingestion program used as a wrapper object to execute an $\mathrm{R}$ program. Should anyone wish to benchmark a method coded in another language, $\mathrm{R}$ could then be used as a script language to execute the given program by invoking a System Command. A list of R packages installed on the docker image is as well provided. Users need: (i) to register to DECONbench on the participate tab and to download the starting kit and the public datasets; (ii) to develop an algorithm according to DECONbench guidelines; (iii) to submit their code (as a zip file) in the participate tab. Submitted algorithms are evaluated on DECONbench datasets and benchmarked with the other baseline methods. Users should note that methods relying on stochastic algorithms will give slightly variable performance on each run, 


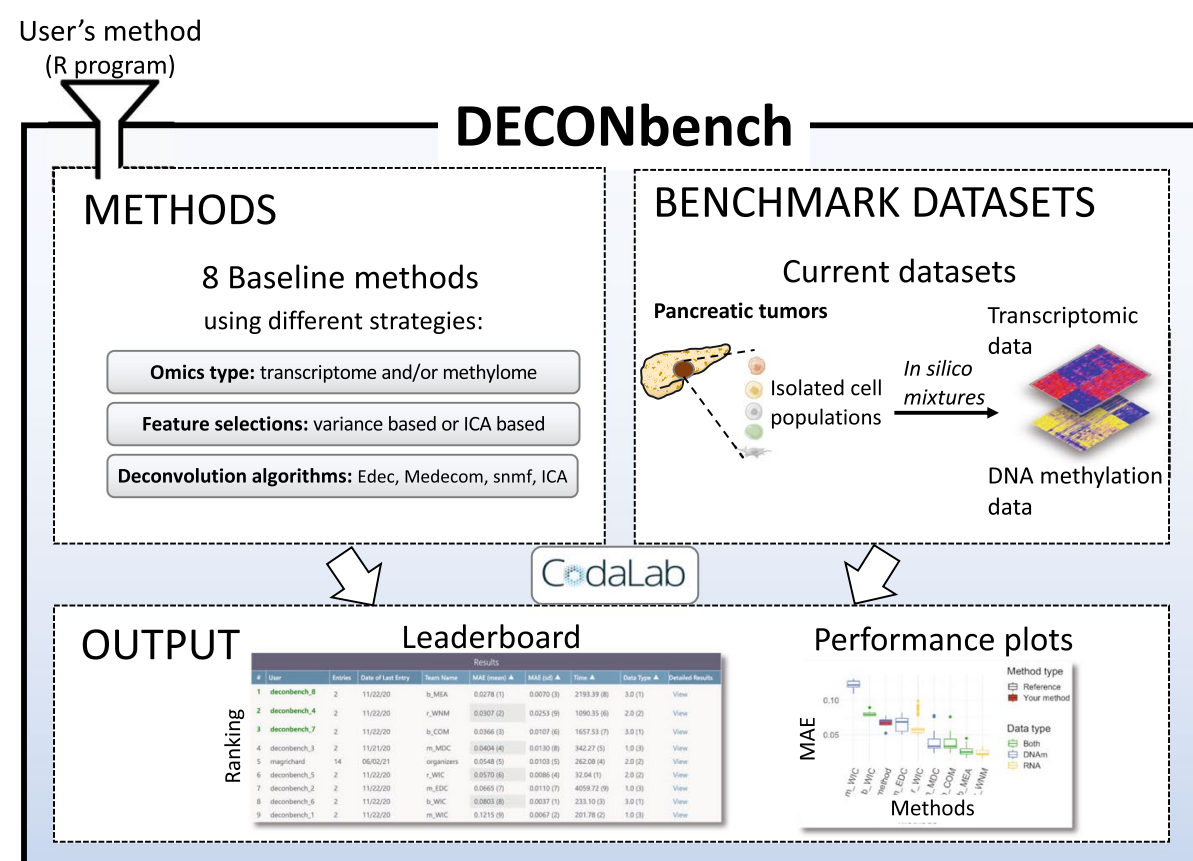

Fig. 1 Overview of the DECONbench platform. The platform proposes a set of 8 baseline deconvolution methods and benchmark datasets consisting of paired methylome and transcriptome of in silico mixtures from pancreatic tumors. The platform releases the performance of each method on a leaderboard and provides plots for deeper evaluation. New methods are automatically compared to the existing ones

unless an initialization is specified in the source code. Resulting scores appear on the leaderboard and a fact sheet is edited summarizing the performances. Importantly, users can choose whether they want their algorithm to be public or private.

\section{Results}

\section{Provided benchmark datasets}

We have generated paired transcriptome and methylome benchmarking datasets from primary cells from pancreatic tumors and sorted cells from public datasets (Fig. 2). Gold standard heterogeneous samples were simulated using mixtures of individual cell populations (fibroblast, immune cells, normal epithelial cells and cancer cells, see "Methods" section). Exact sample compositions are not accessible to the users. Participants are facing a deconvolution problem to solve the following model: $D=T A$, with $D$ the complex matrix of molecular profiles measured on heterogeneous samples; $T$, a reference matrix of cell-type specific molecular profiles; and $A$, a proportion matrix of cell-type abundance in each sample. The aim of the competition is to find the best estimate of the proportion matrix $A$. Methods are evaluated on their accuracy to estimate the cell-type proportions per sample from transcriptome and/or methylome heterogeneous profiles. The discriminating metric is the mean absolute error (MAE, see "Methods" section) between the estimate and the ground truth. 


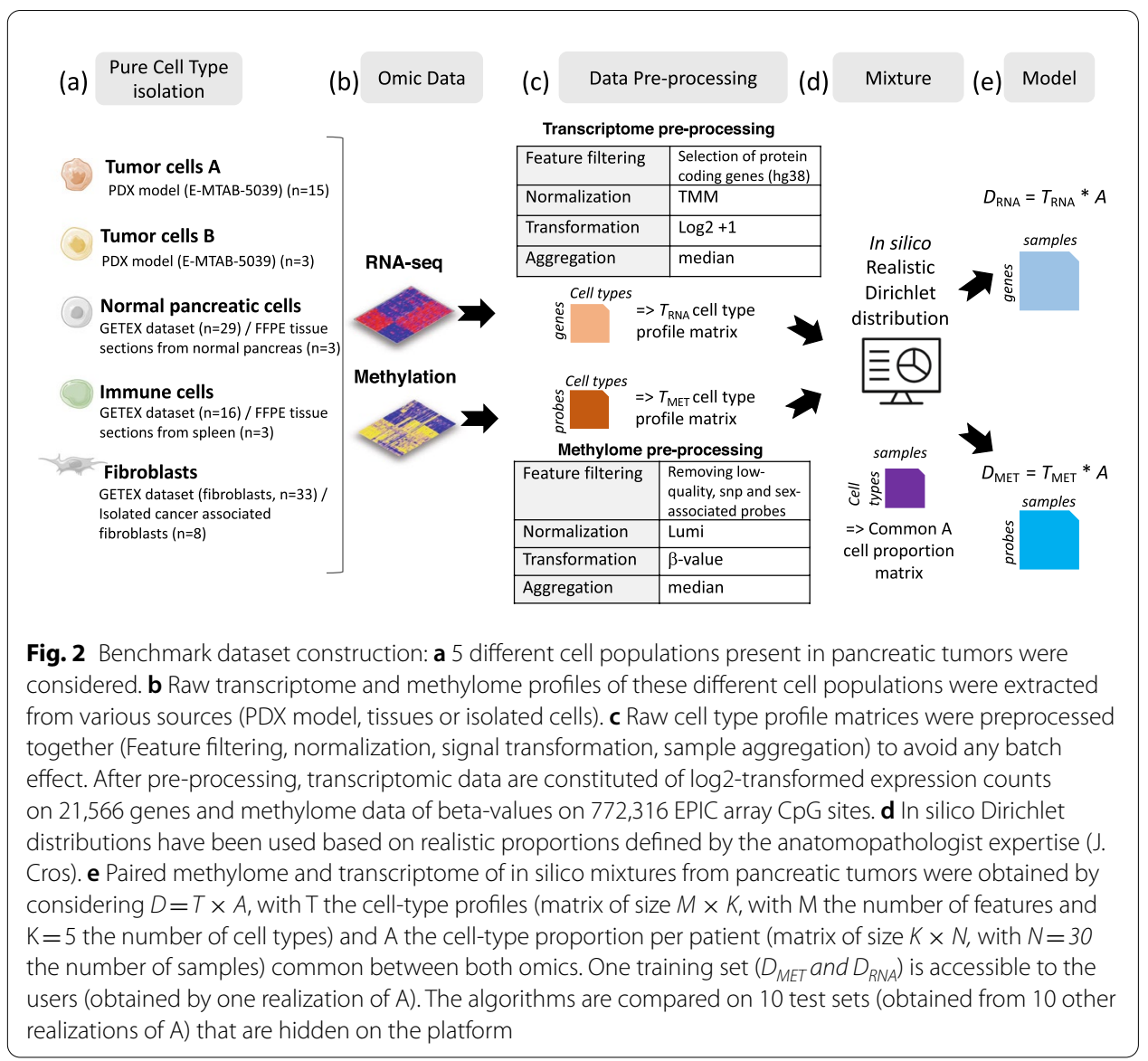

\section{Selection of baseline methods from a data challenge}

We used these unreleased benchmark datasets in a data challenge aiming at inferring cell-type proportions from a cancer dataset including both transcriptome and methylome profiles (https://tinyurl.com/hadaca2019). Baseline methods provided on DECONbench were collectively designed, tested and implemented during the challenge. They are composed of two steps: first, we operate a feature selection process to reduce the dimensions of the dataset, second, we apply a deconvolution algorithm. These algorithms consist of various statistical tools already published, based on unsupervised source separation approaches: ICA-based (Independent Component Analysis) [23-25] or NMF-based (Non-negative Matrix Factorization) $[8,9,26]$. Each baseline method was designed to be applied either on single-omic (see Table 1, Data type "RNA" or "DNAm") or in an integrated fashion on both the transcriptome and the methylome dataset (see Table 1, Data type "both" and Multiomic integration strategy). As baseline on DECONbench, we implemented the eight methods that predict the real cell proportions with the highest accuracy (i.e. lowest MAE between the estimate and the ground truth) (Table 1). All baseline methods source code are publicly accessible on the platform. 


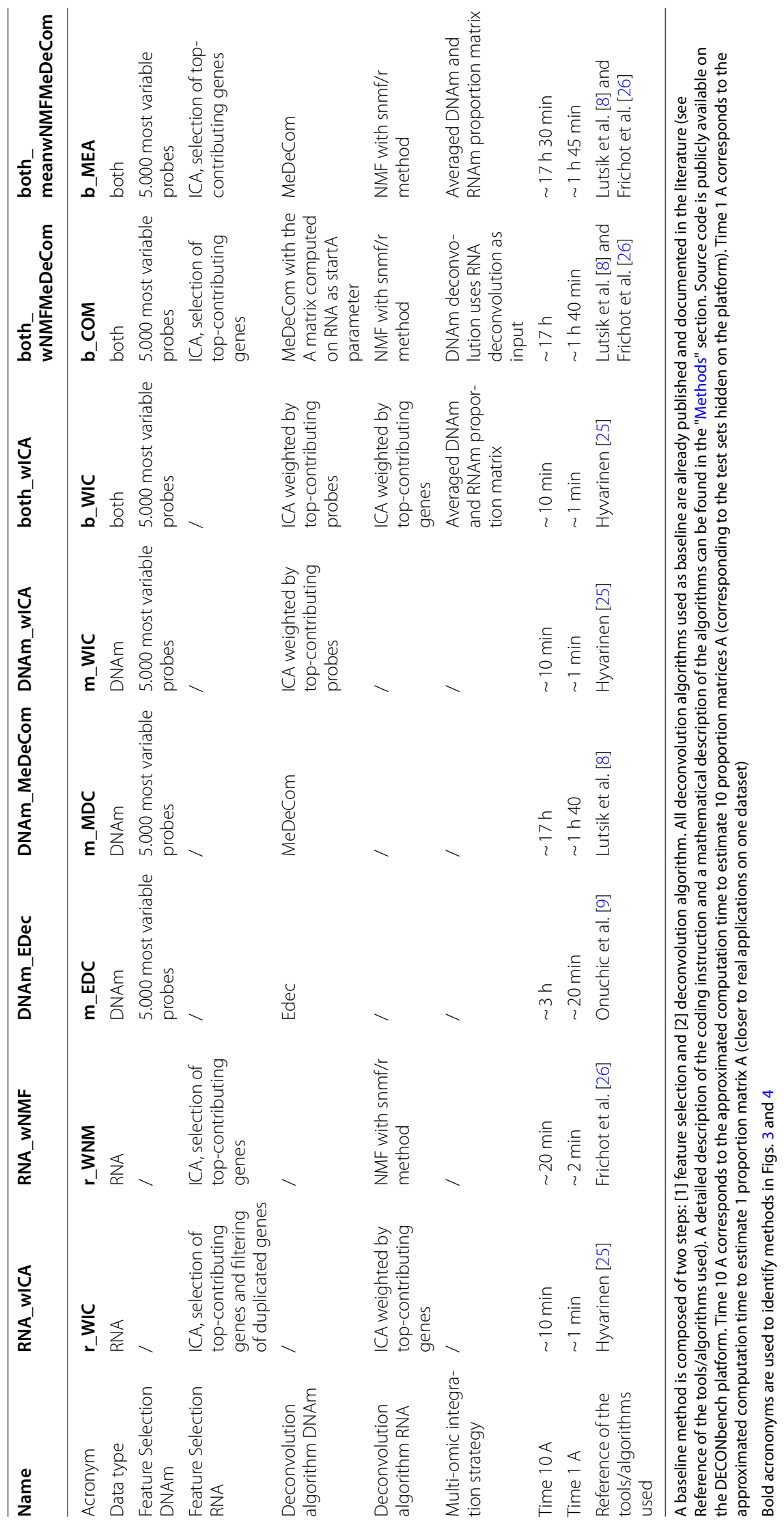




\section{Performance of the baseline single-omic methods}

We run all the baseline methods on 10 different simulated datasets and computed the corresponding MAEs (Fig. 3). The best algorithms based on single-omic datasets were the r_WNM method for RNA-based data (mean MAE of 0.024) and the m_MDC method for DNAm-based data (mean MAE of 0.038). Both are NMF-based algorithms, details on the methods can be found in the "Methods" section. DECONbench provides also the computing time for each method, as an indicator of algorithms optimization. It is worth underlying that the computation time of m_MDC algorithm is significantly higher than the other DNAm-based methods we explored, suggesting that even high performance single-omic algorithms might be further optimized.

\section{Performance of the integrative multi-omics methods}

Next, we tested basic multi-omic approaches averaging the results of single-omic methods: (i) the b_WIC method averages the proportion matrices given by the independent applications of independent component analysis (ICA) based deconvolution approach to transcriptome and methylome data, (ii) the b_MEA method computes an average proportion matrix from the output of the two best single-omic methods r_WNM and $\mathrm{m}_{-}$MDC. Averaging the ICA based approaches (b_WIC) gave intermediate performances (multi-omic accuracy equivalent to the mean of single-omic

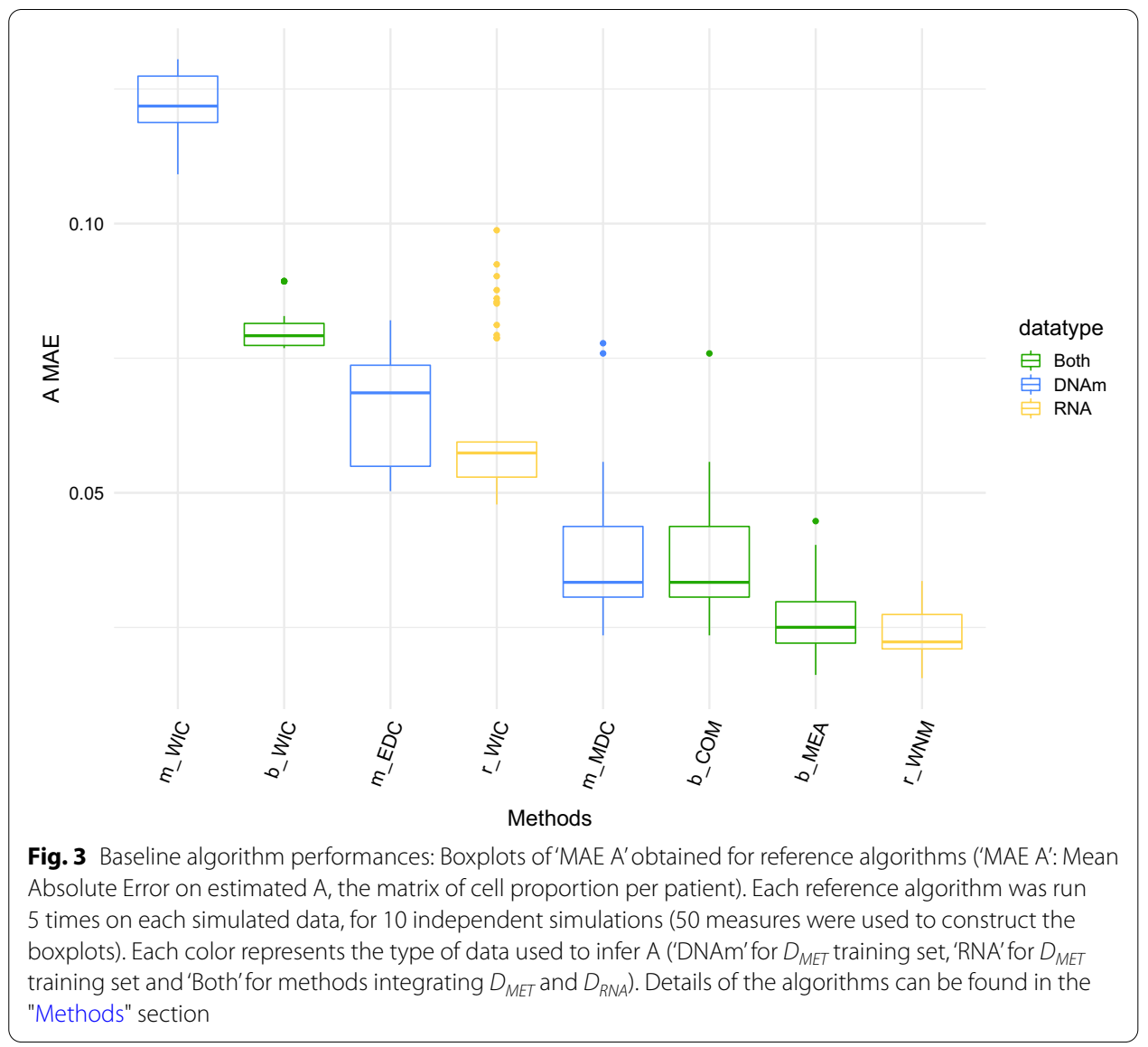


accuracies). Similarly, we did not observe increased performances when averaging the predicted proportion matrices of the two best methods (b_MEA).

We also proposed an integrative method (b_COM) based on transcriptome and methylome data. The best performing methylome-based method ( $\left.m \_M D C\right)$ relies on the MeDeCom tool which is a NMF-based deconvolution algorithm that performs multiple random initializations of the cell-type proportion matrix. Instead of using random initialization, we initialized the MeDeCom algorithm with the proportion matrix obtained from a NMF-based deconvolution of transcriptome data. Surprisingly, we did not observe a substantial performance improvement when integrating RNA deconvolution output into DNAm deconvolution algorithm (b_COM method, resulted in an average error decrease of $2.12 \%$ compared to $\mathrm{m} \_\mathrm{MDC}$ ). These results highlight the need to further develop new methods to improve integration of multi-omic deconvolution algorithms.

\section{Toward crowdsourced and continuous benchmarking}

As an example of continuous benchmarking, we used DECONbench to assess the performances of two recently evaluated single-omic algorithms in a comprehensive benchmark of reference-based deconvolution pipelines. We selected the Ordinary Least Square (OLS) and Robust Linear Regression (RLR) approaches, which have been shown to be effective in estimating cellular composition of simulated bulk healthy pancreatic transcriptomes [10]. We implemented the methods as recommended by Avila Cobos et al., including the generation of cell-type reference profiles from a pancreatic singlecell dataset [27] (see supplemental information for source code: Additional file 1: Source code). Interestingly, the performance of these methods is not better than the baseline methods, possibly due to the use of healthy pancreatic cells as a reference to estimate the composition of a simulated pancreatic adenocarcinoma (Additional file 1: Figure S1). These results suggest that further optimization should be considered to properly assess the performance of the OLS and RLR methods. This crowdsourced and continuous integration is now made possible thanks to our DECONbench platform.

\section{Conclusion}

The DECONBench platform is a unique opportunity to compare the performance of deconvolution methods on different omics data. It can be used to assess the performance of newly developed methods by applying them on high quality benchmark datasets in a user-friendly fashion. Currently, the accuracy of new methods can be compared with the eight baseline methods that have been included in the benchmarking platform. As compared with previous time-bound comprehensive benchmarks of deconvolution methods (see Avila Cobos et al. [10]), our platform provides the possibility to continuously test and integrate newly developed methods, rather than focusing on an exhaustive comparison of existing tools. The baseline methods and user's methods performances are reported on the leaderboard and on the graphical output of DECONBench (Fig. 4). The source code of the baseline methods can be downloaded directly on the DECONbench platform. The structure of DECONbench is open to evolution. Work is ongoing to generate new benchmark datasets including other omic types that will be added to the platform. In the near future, we plan to expand the usability of DECONbench by 


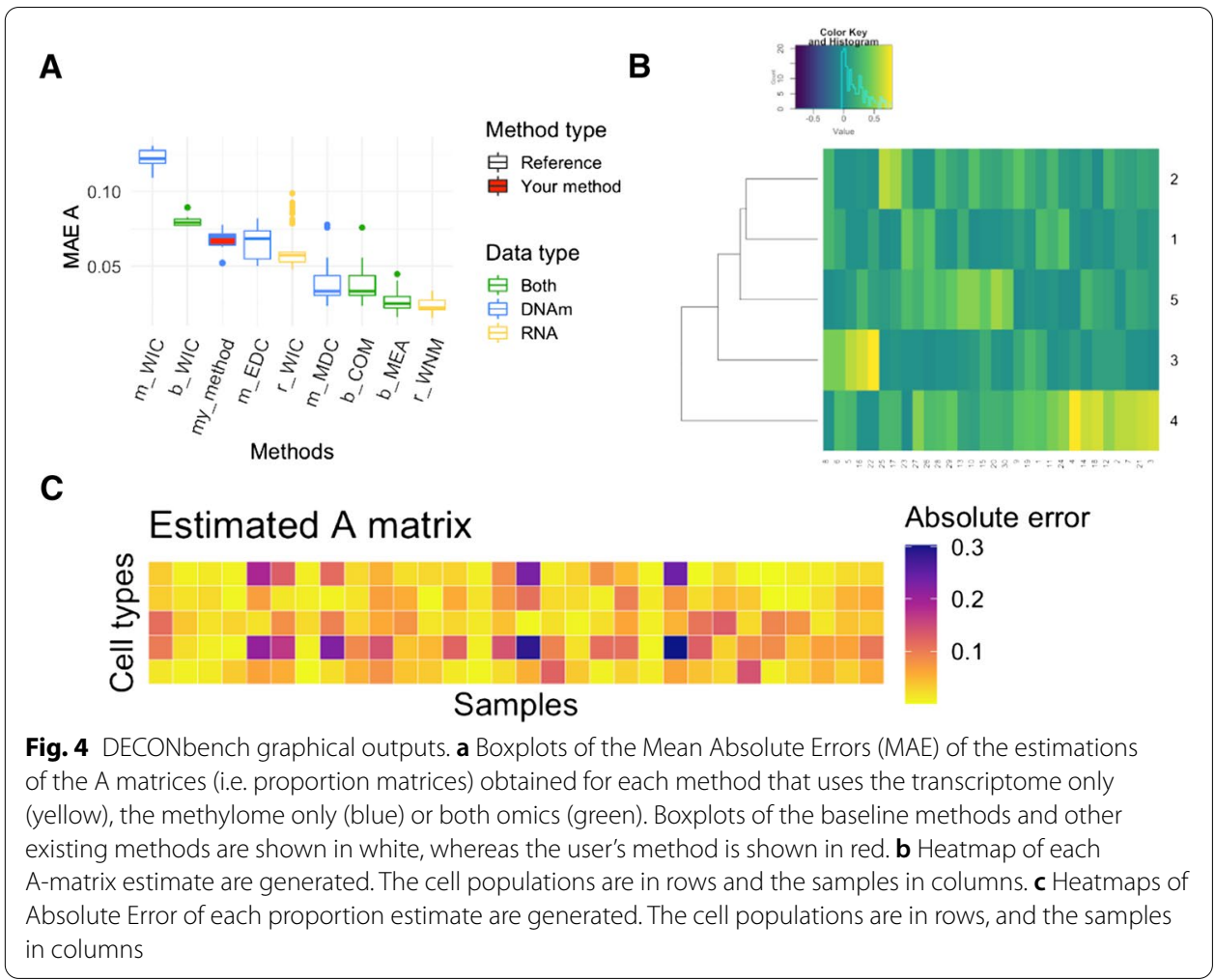

offering the possibility for owners of benchmark datasets to directly upload them on the platform.

DECONbench evaluation framework presents standard benchmark limitations [15, 16], such as the use of artificial in silico simulated data that do not capture the real experimental complexity, or the ranking of the methods based on a single performance metric. We would like to emphasize that MAE as scoring metric is only an imperfect proxy to evaluate quantification of tumor heterogeneity, as it does neither reflect the accuracy of cell-type specific molecular profile prediction (i.e. biological significance of inferred components), nor the correlation of estimated heterogeneity with real clinical outputs (such as prognosis or survival).

Overall, our platform will guide computational biologists to use the best proposed deconvolution algorithms and allow health professionals and biologists to obtain more accurate information regarding the composition of their samples, an important step towards personalized healthcare.

\section{Methods}

\section{Data collection and preprocessing}

For both transcriptome and methylome in silico mixtures, the same five cell types present in pancreatic tumors were considered (Fig. 2a, b): tumor cells A, tumor cells B, normal pancreatic cells, immune cells and fibroblasts. Pure cell type transcriptome profiles were retrieved from the GTEX RNA-seq dataset for the immune and normal pancreatic cell types (https://gtexportal.org/) and a previously published pancreatic tumor patient derived xenograft (PDX) RNA-seq dataset (E-MTAB-5039) for the two tumor cell types 
(total of 96 pure transcriptome profiles with 3 to 33 replicates per cell type) (Fig. 2c). Pure cell type methylation profiles were retrieved from the same samples of the PDX dataset for the two tumor cell types and tissue or isolated cell profiles were used for the microenvironment cell types (total of 32 methylome pure profiles with 3 to 15 replicates per cell type). Transcriptome dataset was restricted to protein coding genes and subjected to TMM normalization using the edgeR R package and log2 transformation. For the methylation data, we used the beta-value DNA methylation scores and removed probes with low-quality, that contained SNPs or located on sex chromosomes. Data were then adjusted for color balance bias and normalized between samples using the SSN (shift and scaling normalization) method using the lumi package functions (Fig. 2c). For both omics, the median of the replicate profiles for each cell type was calculated to compute the $T_{\mathrm{RNA}}$ and $T_{M E T}$ matrices, representing the cell type specific profiles for each omic. The median calculation may prevent underlying germline differences. These matrices were used for the in-silico mixtures, as detailed in the next sections (Fig. 2d, e).

\section{Formulation of the deconvolution problem}

When a sample is constituted of $\mathrm{K}$ cell types, we assume that the level of methylation or gene expression observed in a bulk measurement of this biological sample (containing different cell types) results from a linear mixture of the $\mathrm{K}$ cell-type specific molecular profile weighted by the true cell-types proportions present in the sample. This assumption leads to the following models:

$$
\begin{aligned}
& D_{M E T}=T_{M E T} A \\
& D_{R N A}=T_{R N A} A
\end{aligned}
$$

where $D_{M E T}$ is a $(\mathrm{M} \times \mathrm{N})$ methylation matrix from $\mathrm{N}$ bulk heterogeneous samples with $D_{M E T_{\{m, n\}}}$ the measured measured methylation (beta-value) of the $\mathrm{m} t h \mathrm{CpG}$ site for the $\mathrm{n}$ th sample representing the measured methylation (beta-values) for $\mathrm{N}$ samples; $D_{\mathrm{RNA}}$ is a $(\mathrm{G} \times \mathrm{N})$ gene expression matrix from the same $\mathrm{N}$ bulk heterogeneous samples with $D_{R N A_{\{g, n\}}}$ the measured gene expression (normalized pseudo-log counts) of the gth gene for the $\mathrm{n} t h$ sample; $T_{M E T}$ is an unknown $(\mathrm{M} \times \mathrm{K})$ reference-profile matrix with $T_{M E T_{[m, k\}}}$ representing the average methylation beta-value of CpG site $m$ for the cell-type $k$; $T_{\mathrm{RNA}}$ is an unknown $(\mathrm{G} \times \mathrm{K})$ reference-profile matrix with $T_{R N A_{\{g, k\}}}$ representing the average expression value (normalized pseudo-log counts) of gene $g$ for the cell-type $k$; and $A$ a $(\mathrm{K} \times \mathrm{N})$ matrix representing the cell-type composition of the $N$ heterogeneous samples for $K$ cell types (i.e. the cell-type proportions), with $A_{\{k, n\}}$ the proportion of the $\mathrm{n}$ th sample for the kth cell type. Specifically, the $A$ proportion matrix is shared between the two models, as $D_{M E T}$ and $D_{R N A}$ bulk molecular profiles are measured on the same biological samples. In the methods tested, $A$ is estimated with the following constrain: $\sum_{k=1}^{K} A_{k n}=1$.

\section{Data modeling}

The benchmark simulated bulk molecular profiles are constituted of 10 paired $D_{M E T}$ and $D_{R N A}$ matrices. Simulations are processed as follows: 


\section{Step 1: Simulation of the shared proportion matrices}

The mixture proportions of the matrices A were sampled from a Dirichlet distribution based on realistic biological composition of a pancreatic tumor, with the variation of Dirichlet parameters set to $\alpha_{0}=10$ for global cell composition (fibroblasts, immune, normal epithelial and cancer epithelial), and a variation of Dirichlet parameters set to $\alpha_{0}=1$ for cancer cells subpopulations (cancer basal-like and cancer classic). Exact proportion parameters are kept private to ensure unbiased evaluation of the methods.

\section{Step 2: Simulation of the bulk D bulk matrices}

We use the mathematical models (1) and (2) to simulate the bulk matrices, as previously described in Decamps et al. (2020) [7]. $D_{M E T}$ is a methylation matrix composed of 772,316 methylation values (EPIC array CpG sites) for $\mathrm{N}=30$ samples, $\mathrm{D}_{\text {MET }}$ was constructed as follows: $D_{M E T}=T_{M E T} A$, with $T_{M E T}$ a matrix of $\mathrm{K}=5$ cell type-specific methylation reference profiles (methylation beta-values for each cell type considered: tumor cells $\mathrm{A}$, tumor cells $\mathrm{B}$, normal pancreatic cells, immune cells and fibroblasts), and $A$ a (K $\times N$ ) proportion matrix composed of $K=5$ cell type proportions for each $\mathrm{N}=30$ sample. $D_{\mathrm{RNA}}$ is a transcriptome matrix composed of 21,566 gene expression values (normalized log-2 transformed RNA-seq counts values for each cell type) for $\mathrm{N}=30$ samples. $\mathrm{D}_{\mathrm{RNA}}$ was constructed according to the following model: $D_{R N A}=T_{R N A} A$, with $T_{R N A}$ a matrix of the $\mathrm{K}=5$ cell type-specific transcriptome reference profiles (21,566 gene expression values for each cell type: tumor cells $\mathrm{A}$, tumor cells $\mathrm{B}$, normal pancreatic cells, immune cells and fibroblasts), and $A$ the same $(K \times N)$ proportion matrix used to simulate $D_{\text {MET }}$.

\section{Step 3: Simulation of a technical noise}

We added a generic Gaussian noise on each bulk simulated matrix using the following parameters: $\mathrm{mu}=0$ and $\mathrm{sd}=0.05$.

\section{Step 4: Replication of the simulations}

To ensure robustness of the method's evaluation, we generated 10 replications of paired $D_{M E T}$ and $D_{R N A}$ matrices, using independent simulation of A proportions matrices. For each pair of $D_{M E T}$ and $D_{R N A}$ matrices, the same $T_{M E T}$ and $T_{R N A}$ reference matrices were used.

\section{Performance evaluation}

The aim of deconvolution algorithms is to correctly estimate the proportion matrix A. We evaluated algorithm performances by computing the mean absolute error (MAE), as previously described in Decamps et al. (2020) [7]:

$$
\mathrm{MAE}=\frac{\sum_{\mathrm{n}=1}^{\mathrm{N}} \sum_{\mathrm{k}=1}^{\mathrm{K}}\left|\mathrm{Aest}_{\mathrm{nk}}-\operatorname{Areal}_{\mathrm{nk}}\right|}{\mathrm{NK}}
$$

One training set $\left(D_{M E T}\right.$ and $\left.D_{R N A}\right)$ is publicly available (the $A, T_{R N A}$ and $T_{M E T}$ matrices used for compute $D_{M E T}$ and $D_{R N A}$ matrices remain private, as they are directly involved in performance evaluation). The algorithms are evaluated on 10 test sets $\left(D_{M E T}\right.$ and $D_{R N A}$ ), obtained from 10 independent realizations of A, given the simulation models $D_{M E T}=T_{M E T} A$ and $D_{R N A}=T_{R N A} A$. These test sets are hidden on the platform to avoid 
overfitting. During evaluation of baseline algorithms, each algorithm was run 5 times on each simulated set of data, to account for randomness in algorithm outputs.

\section{Description of the baseline methods}

The baseline methods we propose here are wrappers of already published unsupervised deconvolution algorithms (ICA-based or NMF-based). We assume here that A, $T_{M E T}$ and $T_{R N A}$ are unknown and need to be estimated, either independently (single-omic pipelines) or integratively (double-omic pipelines). Before deconvolution, we systematically apply a pre-treatment step of dimensionality reduction based on feature selection. All baseline methods source code is downloadable on the DECONbench platform.

All baselines relies on unsupervised deconvolution algorithms, which consists in solving $D=T A$, either by ICA-based (i) or NMF-based (ii) approaches. (i) ICA-based approaches ( $r_{-}$WIC, $\mathrm{m}_{-}$WIC and $b_{-}$WIC) consist of minimizing mutual information of sources by defining independent components. It is based on the fixed-point FastICA algorithm developed by Aapo Hyvärinen [24, 25]. (ii) NMF-based approaches (r_WNM, m_EDC, m_MDC, b_COM, b_MEA) aims to minimizing $\|D-T A\|_{2}$.

\section{RNA_wICA (r_WIC, ICA-based deconvolution on RNA)}

The method RNA_wICA (r_WIC) uses transcriptomic data as input and is based on the ICA algorithm for both feature selection and deconvolution. It relies on the use of the functions "runICA" and "getGenesICA" developed by P. Nazarov (sablab.net/ scripts/LibICA.r) and the deconica R package [23].

STEP1: feature selection For the ICA-based feature selection, the function "runICA" is run at first with the parameters ncomp $=10$ and ntry $=50$. Then, the function "getGenesICA" selects top-contributing genes with a FDR of 0.2, the feature selection is done on these contributing genes belonging to a component having an average stability greater than 0.8 . Finally, duplicated genes are removed.

STEP2: deconvolution First, we perform FastICA unsupervised deconvolution (deconica::run_fastica is run with the parameters overdecompose $=$ FALSE and n.comp $=5$; remaining parameters are set to default). Second, we compute the abundance of the identified components, using the weighted-mean of the 30-top genes of each Independent Component (IC), in each sample as, a surrogate of the component signal. The 30 most important genes of each ICA component are extracted by the function deconica::generate_markers with the parameter return = "gene.ranked". These genes are used to weight the component scores in each patient (the weighted-score of a given IC in patient $p$ corresponds to the weighted mean expression of the 30-top genes on that component. We used, in the function deconica::get_scores, the log counts of the ICA as "df" parameter, the list of 30 genes as "markers.list" parameter, and the parameter summary $=$ "weighted.mean". Finally, the estimated proportions are calculated from the inferred weighted-score with the function deconica::stacked_proportions_plot on the transpose of the deconica::get_scores output. 
DNAm_wICA ( $m \_$WIC, ICA-based deconvolution on DNAm)

The method DNAm_wICA (m_WIC) uses DNA methylation data as input.

STEP1: feature selection It has no feature selection step.

STEP2: deconvolution The deconvolution step is based on ICA, similarly to what was described for the second step of RNA_wICA, but applied on the DNA methylation matrix.

both_wICA (b_WIC. ICA-based deconvolution on RNAD and DNAm)

The method both_wICA (b_WIC) combines transcriptomics and DNA methylation information.

STEP1: feature selection It has no feature selection step.

STEP2: deconvolution The deconvolution is in two steps, one on each data type. The transcriptomics and DNA methylation data are separately deconvoluted with the same deconvolution step as in $r_{-}$WIC and $m_{-}$WIC respectively to estimate $\mathrm{A}_{\mathrm{MET}}$ and $\mathrm{A}_{\mathrm{RNA}}$.

STEP3: integration Finally, the mean of both $\mathrm{A}_{\mathrm{MET}}$ and $\mathrm{A}_{\mathrm{RNA}}$ estimated proportion matrices is computed as the final method output. To compute the average, the cell types of the both deconvolution matrices are matched by iteration. The cell types of the methylation result matrix are reordered 1000 times, and the one that best correlates with the transcriptomic result matrix is kept.

\section{RNA_wNMF ( $r$ _WNM, NMF-based deconvolution on RNA)}

The method RNA_wNMF (r_WNM), is a two step-approach that uses transcriptomic data as input.

STEP1: feature selection The first step uses ICA to perform a feature selection as described for RNA_wICA, although duplicated genes are kept. This step therefore allows genes that contribute to several components to be present several times in the data.

STEP2: deconvolution The deconvolution is based on sparse NMF and leastsquares optimization to minimize $\|D-T A\|_{2}$ [26]. It is called by the NMF::nmf function, with the parameter method $=$ "snmf $/ \mathrm{r}$ ".

\section{DNAm_EDec (m_EDC, NMF-based deconvolution on DNAm)}

STEP1: feature selection The method DNAm_EDec (m_EDC), uses DNA methylation data as input and follows the pipeline implemented in the $\mathrm{R}$ package medepir [7]. The feature selection is performed by medepir::feature_selection for keeping highly variable probes (5000 most variable probes). 
STEP2: deconvolution The NMF-based algorithm of the method EDec [9] is used for the deconvolution part, with the function medepir::Edec and all the selected probes as "infloci" parameter. The algorithm consist in minimizing the error term $\|D-T A\|_{2}$ with constraints on methylation values: $0 \leq A \leq 1$ and $0 \leq T \leq 1$ and constraints on proportions $\sum_{k-1}^{K} A_{k n}=1$ where $A_{k n}$ is the proportion of the $n_{t h}$ sample for the $k_{t h}$ cell type.

\section{DNAm_MeDeCom (m_MDC, NMF-based deconvolution on DNAm)}

STEP1: feature selection The method DNAm_MeDeCom (m_MDC), uses DNA methylation data as input and is based on the pipeline of the $\mathrm{R}$ package medepir. The feature selection is performed as for DNAm_EDec above to select the 5000 most variable probes.

STEP2: deconvolution The deconvolution step, however, uses the MeDeCom R package [8]. It is run with the function MeDeCom::runMeDeCom, with the lambda parameter set to 0.01. As EDec implementation of NMF algorithm, MeDeCom algorithm consists in minimizing the error term $\|D-T A\|_{2}$ with constraints on methylation values: $0 \leq A \leq 1$ and $0 \leq T \leq 1$; and constraints on proportions $\sum_{k-1}^{K} A_{k n}=1$ where $A_{k n}$ is the proportion of the $n_{t h}$ sample for the $k_{t h}$ cell type. It also uses a regularization function that favors methylation values close to 0 or 1 .

\section{both_wNMFMeDeCom (b_COM, NMF-based deconvolution on RNA and DNAm)}

The method both_wNMFMeDeCom (b_COM) combines transcriptomics and DNA methylation information. It is the combination of the two methods RNA_wNMF and DNAm_MeDeCom. The method $r_{-}$WNM is first applied to the RNAseq matrix.

STEP1: feature selection The DNA methylation matrix is pre-treated as described in the $\mathrm{m} \_$MDC method, with the selection of 5000 most variable probes.

STEP2-3: deconvolution-integration Finally, the MeDeCom algorithm is run on the DNAm data, with the result of $r_{-}$WNM as the initialization parameter startA.

\section{both_meanwNMFMeDeCom (b_MEA, NMF-based deconvolution on RNA and DNAm)}

The method both_meanwNMFMeDeCom (b_MEA), which integrates transcriptomics and DNA methylation, applies $r_{-}$WNM to the transcriptomics matrix, m_MDC to the DNA methylation matrix.

STEP1: feature selection Feature selection is performed on $\mathrm{D}_{\mathrm{MET}}$ and $\mathrm{D}_{\mathrm{RNA}}$ matrices as described in r_WNM and $\mathrm{m}_{-}$MDC sections.

STEP2: deconvolution Deconvolution is performed on $\mathrm{D}_{\mathrm{MET}}$ and $\mathrm{D}_{\mathrm{RNA}}$ matrices as described in $r_{-} W N M$ and $m_{-}$MDC sections to estimate $A_{M E T}$ and $A_{R N A}$ matrices.

STEP3: integration We computed the mean of the two estimated $\mathrm{A}_{\mathrm{MET}}$ and $\mathrm{A}_{\mathrm{RNA}}$ matrices, similarly to b_WIC. 


\title{
Availability and requirements
}

\author{
Project name: DECONbench \\ Project home page: https://competitions.codalab.org/competitions/27453 \\ Operating system(s): Linux (CodaLab platform)/Debian (DECONbench) \\ Programming language: Python (CodaLab platform)/R (DECONbench) \\ Other requirements: none \\ License: Apache 2.0 (CodaLab platform)/CeCILL (DECONbench) \\ Any restrictions to use by non-academics: none
}

\section{Abbreviations}

DNAm: DNA methylation; FDR: False discovery rate; ICA: Independent component analysis; MAE: Mean absolute error; NMF: Non-negative matrix factorization; sd: Standard deviation; var: Variance.

\section{Supplementary Information}

The online version contains supplementary material available at https://doi.org/10.1186/s12859-021-04381-4.

Additional file 1: Figure S1: DECONbench benchmark of OLS and RLR methods: an example of graphical outputs of new contributions to the benchmark. Source code.

\section{Acknowledgements}

We thank all members of the HADACA consortium for helpful discussion and contributions during the HADACA data challenge 2nd edition (November 2019, Aussois, France). We also thank Daniel Jost and the members of the BCM team for inspiring discussions during regular joint group meetings. We are grateful to the Codalab data challenge open source platform. The authors gratefully acknowledge the EpiMed core facility for their support and assistance in this work. This work is part of the national program Cartes d'Identité des Tumeurs supported by the Ligue Nationale Contre le Cancer. Where authors are identified as personnel of the International Agency for Research on Cancer/World Health Organization, the authors alone are responsible for the views expressed in this article and they do not necessarily represent the decisions, policy or views of the International Agency for Research on Cancer/World Health Organization.

\section{HADACA (Health Data Challenge) Consortium}

Nicolas Alcala ${ }^{6}$, Alexis Arnaud ${ }^{2}$, Francisco Avila Cobos ${ }^{7}$, Luciana Batista ${ }^{8}$, Anne-Françoise Batto ${ }^{9}$, Yuna Blum ${ }^{3}$, Florent Chuffart $^{10}$, Jérôme Cros $^{5}$, Clémentine Decamps ${ }^{1}$, Lara Dirian ${ }^{11}$, Daria Doncevic ${ }^{12}$, Ghislain Durif ${ }^{13}$, Silvia Yahel Bahena Hernandez ${ }^{14}$, Milan Jakobi ${ }^{10}$, Rémy Jardillier $^{15}$, Marine Jeanmougin ${ }^{16}$, Paulina Jedynak ${ }^{10}$, Basile Jumentier ${ }^{1}$, Aliaksandra Kakoichankava ${ }^{17}$, Maria Kondili ${ }^{18}$, Jing Liu ${ }^{19}$, Tiago Maie ${ }^{20}$, Jules Marécaille ${ }^{11}$, Jane Merlevede ${ }^{21}$, Maxime Meylan ${ }^{3,22}$, Petr Nazarov $^{23}$, Kapil Newar ${ }^{1}$, Karl Nyrén ${ }^{14}$, Florent Petitprez ${ }^{3}$, Claudio Novella Rausell ${ }^{14}$, Magali Richard ${ }^{1}$, Michael Scherer ${ }^{24}$, Nicolas Sompairac ${ }^{21}$, Katharina Waury ${ }^{14}$, Ting Xie ${ }^{25}$ and Markella-Achilleia Zacharouli ${ }^{14}$

1. Laboratory TIMC-IMAG, UMR 5525, Univ. Grenoble Alpes, CNRS, Grenoble, France. 2. Data Institute, Univ. Grenoble Alpes, Grenoble, France. 3. Programme Cartes d'Identité des Tumeurs (CIT), Ligue Nationale Contre le Cancer, Paris, France. 4. INSERM U1068 CRCM, Marseille, France. 6. Section of Genetics, International Agency for Research on Cancer (IARC-WHO), Lyon, France. 7. Center for Medical Genetics Ghent, Department of Biomolecular Medicine, Ghent University, Ghent, Belgium. 8. Innate Pharma, Marseille, France. 9. Equipe Cancer et Immunité- INSERM Centre de Recherche des Cordeliers, Paris, France. 10. Institute for Advanced Biosciences, CNRS UMR 5309, Inserm, U1209, Univ. Grenoble Alpes, F-38700 Grenoble, France. 11. Verteego, Paris, France. 12. Health Data Science Unit, BioQuant Center and Medical Faculty Heidelberg, Germany. 13. Université de Montpellier, CNRS, IMAG UMR 5149, Montpellier, France. 14. Uppsala University, SE-751 05, Uppsala, Sweden. 15. University Grenoble Alpes, CEA, INSERM, IRIG, Biology of Cancer Infection UMR_S 1036, 38000 Grenoble, France \& University Grenoble Alpes, CNRS, Grenoble INP, GIPSA-lab, Institute of Engineering University Grenoble Alpes, 38000 Grenoble, France. 16. Department of Molecular Oncology, Institute for Cancer Research, Oslo University Hospital, The Norwegian Radium Hospital - Oslo, Norway. 17. Vitebsk State Medical University \& NatiVita, Vitebsk, Belarus. 18. Centre de Recherche de St. Antoine, Paris, AP-HP. 19. Institut Curie, PSL Research University, Sorbonne Universités, UPMC Université Paris 06, CNRS, UMR144, Equipe Labellisée Ligue contre le Cancer, 75005 Paris, France. 20. Institute for Computational Genomics, Joint Research Center for Computational Biomedicine, RWTH Aachen University Medical School, Aachen, Germany. 21. Institut Curie, PSL Research University, Mines Paris Tech, Inserm, U900, F-75005, Paris, France. 22. INSERM U1138 Centre de Recherche des Cordeliers, France. 23. Quantitative Biology Unit, Luxembourg Institute of Health, L-1445 Strassen, Luxembourg. 14. Uppsala University, SE-751 05, Uppsala, Sweden. 24. Department of Genetics/Epigenetics, Saarland University, Saarbrücken, Germany. 25. Centre de Recherche en Cancérologie de Toulouse, Inserm UMR 1037, F-31037, Toulouse, France. 


\section{Authors' contributions}

$M R$ and $Y B$ conceived and designed the project. MR, YB, CD, AA, FP implemented the DECONbench platform. JC, AB, LA prepared the tissue samples and extracted the biological material for the benchmark dataset generation. IG and SE contributed to the development of the platform. MR, YB, CD, AA, FP, MA, RN, RT, AdR contributed to the benchmark dataset generation. HADACA consortium proposed and implemented the reference methods. MR, YB, CD, AA, FP wrote the manuscript. All authors read and approved the final manuscript.

\section{Funding}

The research leading to these results was supported by Univ. Grenoble-Alpes via the Grenoble Alpes Data Institute [MR, AA] (ANR-15-IDEX-02), EIT Health Campus HADACA and COMETH programs [MR, YB], activities 19359 and 20377 and the Ligue Nationale Contre le Cancer. Other fundings: South-Eastern Norway Regional Health Authority (project number 2019030 [MJ]), European IMI IMMUCAN project [NS], European Union's Horizon 2020 program (Grant 826121, iPC project, $[J M, F A C])$. This article did not receive specific sponsorship in the design of the study, analysis, interpretation of data and in writing the manuscript.

\section{Availability of data and materials}

DECONbench is hosted on the open source Codalab competition platform. It is freely available at: https://competitions. codalab.org/competitions/27453. Further documentation (online demo) is available at: https://deconbench.github.io/.

\section{Declarations}

\section{Ethics approval and consent to participate}

Not applicable.

\section{Consent for publication}

Not applicable.

Competing interests

The authors declare that they have no competing interests.

\section{Author details}

${ }^{1}$ Laboratory TIMC-IMAG, UMR 5525, CNRS, Univ. Grenoble Alpes, Grenoble, France. ${ }^{2}$ Data Institute, Univ. Grenoble Alpes, Grenoble, France. ${ }^{3}$ Programme Cartes d'Identité des Tumeurs (CIT), Ligue Nationale Contre le Cancer, Paris, France. ${ }^{4}$ Universitat de Barcelona and Computer Vision Center, Barcelona, Spain. ${ }^{5}$ LISN (INRIA/CNRS), Université Paris-Saclay, Gif-sur-Yvette, France. ${ }^{6}$ INSERM U1068 CRCM, Marseille, France. ${ }^{7}$ Dpt of Pathology, Beaujon Hospital, Univ. Paris-INSERM U1149, Clichy, France. ${ }^{8}$ IGDR UMR 6290, CNRS, Université de Rennes 1, Rennes, France.

Received: 30 October 2020 Accepted: 20 September 2021

Published online: 02 October 2021

\section{References}

1. Avila Cobos F, Vandesompele J, Mestdagh P, De Preter K. Computational deconvolution of transcriptomics data from mixed cell populations. Bioinformatics. 2018;34:1969-79.

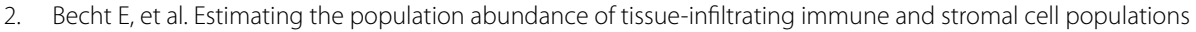
using gene expression. Genome Biol. 2016;17:1-20.

3. Nazarov PV, et al. Deconvolution of transcriptomes and miRNomes by independent component analysis provides insights into biological processes and clinical outcomes of melanoma patients. BMC Med Genomics. 2019;12:1-17.

4. Blum Y, et al. Dissecting heterogeneity in malignant pleural mesothelioma through histo-molecular gradients for clinical applications. Nat Commun. 2019;10:1333.

5. Steen CB, Liu CL, Alizadeh AA, Newman AM. Profiling cell type abundance and expression in bulk tissues with CIBERSORTx. Methods Mol Biol Clifton NJ. 2020;2117:135-57.

6. Houseman EA, Molitor J, Marsit CJ. Reference-free cell mixture adjustments in analysis of DNA methylation data. Bioinformatics. 2014;30:1431-9.

7. HADACA Consortium, et al. Guidelines for cell-type heterogeneity quantification based on a comparative analysis of reference-free DNA methylation deconvolution software. BMC Bioinform. 2020;21:16.

8. Lutsik P, et al. MeDeCom: discovery and quantification of latent components of heterogeneous methylomes. Genome Biol. 2017;18:1-20.

9. Onuchic $V$, et al. Epigenomic deconvolution of breast tumors reveals metabolic coupling between constituent cell types. Cell Rep. 2016;17:2075-86.

10. Avila Cobos F, Alquicira-Hernandez J, Powell JE, Mestdagh P, De Preter K. Benchmarking of cell type deconvolution pipelines for transcriptomics data. Nat Commun. 2020;11:5650.

11. Jin H, Liu Z. A benchmark for RNA-seq deconvolution analysis under dynamic testing environments. Genome Biol. 2021:22:102.

12. White BS, et al. Abstract 1690: A tumor deconvolution DREAM challenge: inferring immune infiltration from bulk gene expression data. Cancer Res. 2019;79:1690-1690.

13. Norel R, Rice JJ, Stolovitzky G. The self-assessment trap: can we all be better than average? Mol Syst Biol. $2011 ; 7: 537$.

14. Buchka S, Hapfelmeier A, Gardner PP, Wilson R, Boulesteix A-L. On the optimistic performance evaluation of newly introduced bioinformatic methods. Genome Biol. 2021;22:1-8.

15. Mangul S, et al. Systematic benchmarking of omics computational tools. Nat Commun. 2019;10:1393. 
16. Marx V. Bench pressing with genomics benchmarkers. Nat Methods. 2020;17:255-8.

17. Krusche P, et al. Best practices for benchmarking germline small-variant calls in human genomes. Nat Biotechnol. 2019;37:555-60

18. Pratapa A, Jalihal AP, Law JN, Bharadwaj A, Murali TM. Benchmarking algorithms for gene regulatory network inference from single-cell transcriptomic data. Nat Methods. 2020;17:147-54.

19. Puleo F, et al. Stratification of pancreatic ductal adenocarcinomas based on tumor and microenvironment features. Gastroenterology. 2018;155:1999-2013.e3.

20. Maurer $C$, et al. Experimental microdissection enables functional harmonisation of pancreatic cancer subtypes. Gut. 2019:68:1034-43.

21. Collisson EA, Bailey P, Chang DK, Biankin AV. Molecular subtypes of pancreatic cancer. Nat Rev Gastroenterol Hepatol. 2019:16:207-20

22. Ellrott $\mathrm{K}$, et al. Reproducible biomedical benchmarking in the cloud: lessons from crowd-sourced data challenges. Genome Biol. 2019;20:195

23. Czerwinska U. UrszulaCzerwinska/DeconICA: DeconICA first release. Zenodo. 2018. https://doi.org/10.5281/zenodo, 1250070.

24. fastICA: FastICA algorithms to perform ICA and projection pursuit. https://CRAN.R-project.org/package=fastICA.

25. Hyvarinen A. Fast and robust fixed-point algorithms for independent component analysis. IEEE Trans Neural Netw. 1999;10:626-34.

26. Frichot E, Mathieu F, Trouillon T, Bouchard G, François O. Fast and efficient estimation of individual ancestry coefficients. Genetics. 2014:196:973-83.

27. Baron M, et al. A single-cell transcriptomic map of the human and mouse pancreas reveals inter- and intra-cell population structure. Cell Syst. 2016;3:346-360.e4.

\section{Publisher's Note}

Springer Nature remains neutral with regard to jurisdictional claims in published maps and institutional affiliations.

- fast, convenient online submission

- thorough peer review by experienced researchers in your field

- rapid publication on acceptance

- support for research data, including large and complex data types

- gold Open Access which fosters wider collaboration and increased citations

- maximum visibility for your research: over $100 \mathrm{M}$ website views per year

At BMC, research is always in progress.

Learn more biomedcentral.com/submissions 